Research Square
Preprints are preliminary reports that have not undergone peer review.

They should not be considered conclusive, used to inform clinical practice, or referenced by the media as validated information.

\title{
Are People with Poor Differentiation of Self at Risk of Developing Eating Disorders? A Study of Jewish and Arab Participants
}

Ora Peleg ( $\sim$ pelegora@gmail.com)

Max Stern Yezreel Valley College https://orcid.org/0000-0002-1257-4066

Orna Tzischinsky

Max Stern Academic College Of Emek Yezreel: Max Stern Yezreel Valley College

\section{Research Article}

Keywords: Eating Disorders, Differentiation of Self, Gender Differences, Jews, Arabs

Posted Date: January 6th, 2022

DOI: https://doi.org/10.21203/rs.3.rs-1221292/v1

License: () (7) This work is licensed under a Creative Commons Attribution 4.0 International License. Read Full License 


\section{Abstract}

Purpose: In light of findings that Israeli Arabs report higher prevalence of eating disorders (EDs) than Israeli Jews and that higher rates of the risk of EDs have been reported by females than males, the first aim of this study was to further investigate the cultural and gender differences in differentiation of self (DoS) and risk of developing EDs among Israeli young adults. The second aim was to examine whether DoS is associated with the risk of EDs.

Methods: Of the 859 participants (670 females, mean age 26.8), 440 were Jewish and 419 were Arab. Participants took the Eating Attitudes Test (EAT-26) and completed the Differentiation of Self-Revised (DSI-R) questionnaire.

Results: Results revealed that Jewish participants had significantly lower diet, bulimia, and total EAT-26 scores than their Arab counterparts, while Jewish female participants had higher dieting and lower emotional cutoff scores than Jewish male participants. In addition, Jews who reported higher levels of risk of EDs showed higher levels of BMI, emotional reactivity, emotional cutoff, and fusion with others. Arabs who reported higher levels of risk of EDs reported higher levels of BMI and emotional cutoff and lower levels of I-position.

Conclusion: People with high risk of EDs may have difficulty maintaining intimate family relationships. In distressing situations, they tend to disconnect rather than share with or gain support from significant others. In each culture, the risk of EDs increases for differently: among the Arab participants, when feelings and needs remain unexpressed; among the Jewish participants, even when they have symbiotic family relationships.

Level of evidence: Level V, cross-sectional descriptive study

\section{Introduction}

Studies have indicated that eating disorders (EDs) have become a global phenomenon over the last two decades [1,2], with a consistent rise in incidences among males and females in both Western [3-7] and non-Western countries (e.g., [8]). Latzer et al., [8] found no significant differences in disturbed eating attitudes (scores above 20) between Arab adolescent boys and girls in Israel. The prevalence of abnormal eating attitudes (scores above 20) was $17.7 \%$ for the full sample, with a breakdown of $16.4 \%$ for the boys and $18.7 \%$ for the girls. According to the scores of the Eating Attitudes Test (EAT-26), the risk of developing EDs among college students is on the rise. In Western countries, the prevalence among college students is about 25\% [9], and in non-Western countries, a prevalence of $11 \%-13 \%$ was found among college students in Malaysia [10,11] and other Asian countries [12], 32.8\% among college students in Morocco [13], 35.4\% among female college students in Saudi Arabia [14], and 25\% among Israeli Arab adolescents (20).

Research in both Western $[7,15,16]$ and non-Western countries $[17,18]$ has pointed to cultural differences in the prevalence of EDs. Several studies conducted in Israel - a country comprised of collectivist and individualist cultures and majority and minority groups [19-23] - have suggested that EDs might be perceived and experienced differently among Arabs and that typical features of the majority group (Jews) cannot always be applied to minority groups (Israeli Arabs) $[24,25]$. Approximately $20.9 \%$ of the Israeli population is Arab, comprising three main religious groups: Muslims, Christians, and Druze [26]. The Arab society in Israel is, in general, undergoing a transition from traditionalism to modernization. These processes are manifested by changes in the economy, education, the status of women, family structure, and by socio-cultural changes and transformations in patterns of coping with social problems. Regarding issues of body image, it is, on the one hand, a conservative society that does not believe in the ideal of thinness; on the other, it is exposed to Western Jewish culture and social media which presents different norms of ideal thinness and nutrition [25]. It was reported that for Muslim respondents and consistent with numerous studies, weight is the largest contributor, whereas for Jews, weight is not a significant predictor. In addition, it was found that for Arab Muslims, selfcriticism scarcely contribute to EDs, while for Israeli Jews, it is a significant predictor of EDs [27]. Although a higher rate of EDs has been found among Israeli Arabs than Israeli Jews [28], to the best of our knowledge, only a few studies conducted in Israel have examined Jewish and Arab adolescents separately. Due to increasing rates of EDs among young adults, and specifically Israeli Arab females [20,21,22], it is important to examine gender and cross-cultural differences within this age group. The present study is aimed to compare the risk of developing EDs between participants from a Western culture (Israeli Jews) and a non-Western culture (Israeli Arabs) living in the same country.

A survey conducted by the World Health Organization showed that dieting behavior is higher among Israeli adolescent girls and boys than among their counterparts in 34 other countries [29]. Latzer et al. [30] found adolescent boys to have lower levels of disordered eating pathology (DEP) than adolescent girls and younger adolescents (grades 8-9) to have higher levels of clinically relevant DEP than older adolescents (grades 10-12). This difference suggests that even at the same developmental stage, adolescent boys are not yet influenced by the same sociocultural standards and expectations as adolescent girls. However, it is also possible that adolescent boys are less sensitive to the cognitive and behavioral experiences than adolescent girls. Participants in the current study comprised young adult males and females.

Young adults with EDs have been found to present high levels of depression and anxiety and increased maladaptive family patterns. Early detection and identification of risk factors of EDs is crucial for preventing complications and increasing recovery [31]. Notwithstanding factors that have been suggested as responsible for increasing the risk of EDs - such as genetic, biological, environmental (media), and psychological (e.g., depression) factors as well as a history of sexual harassment - some recent studies have indicated the need to also detect and examine the contribution of cultural and family factors [32].

In a review describing the role of the family in eating disorders [33], a series of factors have been presented, such as parental pressures, family discord, lack of parental care, depressive symptoms, changing in family structure (e.g., a parent leaving or a step-parent entering the family), low parental contact, and more family criticism about shape and weight. It was claimed that the findings on this topic are inconsistent, and that cross sectional and longitudinal studies yielded several limitations. Thus, for instance, these studies have lacked acceptable statistical power to detect risk factors related to relatively uncommon disorders such as Anorexia Nervosa (AN) and Bulimia Nervosa (BN). 
In light of the methodological shortcomings of retrospective/cross-sectional research, Le grange et al., [33] suggested that family/parenting indices that precede the onset of AN or BN increase risk for psychopathology in general. General risks that interact with inherent and specific sources of biological weaknesses may give shape to specific phenotypes of disordered eating. Therefore, the researchers suggested cautiously considering the results of studies that perceive parents as guilty and responsible for their offspring's EDs and instead focus on involving parents in caring for children with EDs in case it is possible and beneficial.

On the one hand current knowledge refutes the idea that family patterns are either the exclusive or even the primary mechanisms increasing the risk of Eds [e.g., 33]. On the other, a few recent studies examining a specific intergenerational pattern, differentiation of self (DoS), pointed to the importance of this pattern and to the possible detrimental role of parental inaction in relation to EDs among adolescent and adults [e.g. 34 ]. Thus, for example, it was reported that alexithymia and psychological distress mediated the relationships between low DoS and EDs symptoms among adolescents [34], that family patterns were significantly related to the risk of developing EDs [35,36] and that DoS might contribute to the development and severity of EDs [37].

DoS is an important family pattern, shaped by one's family of origin and likely to be a regulating factor of psychological and physical distress. At the interpersonal level, DoS is defined as the ability to balance intimacy with and autonomy from significant others. At the intrapersonal level, it is defined as the ability to balance rational thinking and emotions; poor DoS in the intrapersonal realm may lead to difficulties in recognizing emotional situations and expressing one's own emotions [38]. DoS includes four metrics: emotional reactivity (people with high levels feel overwhelmed when facing stressful situations); I-position (people with high levels can express their desires, thoughts, and needs assertively); emotional cutoff (people with high levels tend to disconnect from people physically or emotionally when they fear intimacy); and fusion with others (people with high levels tend to create dependent relationships with blurred boundaries) [38,39]. The lower the individual's level of emotional reactivity, emotional cutoff, and fusion with others and the higher their level of I-position, the higher their level of DoS.

A number of studies examining DoS have observed gender differences in specific dimensions (although not in the total score). For example, males reported higher levels of I-position and emotional cutoff [40], while females reported higher levels of emotional reactivity and fusion with others [41,42]. Regarding cultural differences, several studies in Israel found differences between Arabs and Jews. For example, Arab Druze mothers reported higher levels of I-position and fusion with others than Jewish mothers [43], and Muslim and Christian Arabs reported higher levels of emotional cutoff, while Jews reported higher levels of emotional reactivity and fusion with others [44].

DoS affects how people cope with distress, anxiety, and frustration. High levels of DoS have been negatively associated with dissatisfaction, anxiety, depression, and alexithymia [45,46] and positively associated with satisfaction with quality of life [47-49]. In a few studies people with higher risk of EDs reported low levels of DoS [40,51] and high levels of distress and alexithymia [52,53]. A recent study of Israeli Arabs and Israeli Jews found cultural and gender differences in EDs and in a few dimensions of DoS [55]. Doba et al. [34] suggested that low levels of DoS may increase confusion between one's own internal emotions and those of others, thereby decreasing the ability to identify emotions and share them with significant others. This, in turn, can lead to a misunderstanding of emotional situations and to distress, which is likely to be expressed through extreme preoccupation with weight or eating [54]. Therefore, DoS is an important factor in understanding the development of psychological distress and risk of EDs.

\section{Research hypotheses}

As seen above, Israeli Arabs reported higher prevalence of EDs and levels of emotional cutoff and lower levels of emotional reactivity and fusion with others than Israeli Jews [19-23], and Israeli Arab females reported higher risk of EDs, emotional reactivity, and fusion with others than males [55,56]. In light of these findings, our first research aim was to further investigate cultural and gender differences in DoS and the risk of EDs among young adults in Israel. Due to differences in DoS between Jews and Arabs, it was assumed that Arabs would report higher risk of EDs and levels of emotional cutoff and lower levels of emotional reactivity and fusion with others than Jews (Hypothesis 1). It was also expected that women would report higher risk of EDs and levels of emotional reactivity and fusion with others than men (Hypothesis 2).

In addition, despite evidence that DoS is a central factor that may increase vulnerability to certain physiological pathologies, such as EDs, by increasing susceptibility to psychological distress [34,50], only a few studies have examined this issue (e.g., among men [37] and among adolescent girls [50]). Given this evidence and the paucity of literature examining the relationship between DoS and EDs among young adults belonging to collectivist and individualist cultures, our second research aim was to determine whether low DoS contributes to the risk of developing EDs among male and female Israeli Jews and Arabs. Specifically, we assumed that the risk of EDs (dieting, bulimia, and food preoccupation, oral control, total EAT-26 score) would be negatively associated with DoS (high emotional reactivity, low I-position, high emotional cutoff, and high fusion with others) (Hypothesis 3).

\section{Methods Participants}

A convenience sample of 1086 non-clinical participants was recruited during the years 2018-2019 via cluster sampling in two colleges located in northern Israel, one with a majority of Jewish students and the other with a majority of Arab students. At the Jewish college, questionnaires were distributed to undergraduate students in the departments of education, economics, social work and computers. At the Arab college, questionnaires were given to undergraduate students in the departments of education, teaching, mathematics and computers. Inclusion criteria were young adults (aged 18-40) with parents who were married (or living together). 
Of the 1086 individuals, we excluded 225 participants who were younger than 18, one respondent who did not indicate gender and another who completed less than half the questionnaire. Thus, the final sample consisted of $859(79 \%)$ participants ( 670 females, 189 males, mean age $26.8, \mathrm{SD}=5.6$, range $18-40$ years; $94.1 \%$ native born). Of them, 440 (51.2\%) were Jewish and 419 were Arab (48.8\%). Mean BMI was 23.85 ; SD $=4.31,17.9 \%$ of the participants were overweight $(25<\mathrm{BMK}<30), 6.4 \%$ were obese $(>30), 4.3 \%$ were underweight $(\mathrm{BMl}<18.5), 413(48 \%)$ had normal weight and the remaining 200 did not report their weight.

\section{Instruments}

A Demographic Questionnaire was constructed for the purpose of the current study, including details regarding age, BMl, gender, ethnicity, etc.

The Differentiation of Self Inventory-Revised (DSI-R, [39,42], translated to Hebrew [57,58], was used to examine levels of differentiation of self. The DSI-R includes 46 items divided into four subscales: emotional reactivity, I-position, emotional cutoff and fusion with others (sample item: "I'm overly sensitive to criticism"). Participants rank each item on a scale of 1 (not at all like me) to 6 (very much like me). Subscale scores are calculated by averaging the mean scores of the items in each subscale. Greater differentiation of self is indicated by lower means for emotional reactivity, emotional cutoff and fusion with others, and by higher means for I-position. The emotional reactivity and emotional cutoff subscales had good reliability (Cronbach's alpha=.871, .830, respectively), while I-position and fusion with others subscales had acceptable reliability (Cronbach's alpha=.781, .731, respectively). The instrument was validated in previous research conducted among Israeli Jews and Arabs [44].

The Eating Attitude Test (EAT-26; [59]) is widely used as a screening instrument measuring risk of developing eating attitudes in large non-clinical populations. This measure describes disordered attitudes and behaviors related to eating, and can identify those requiring additional assessment for EDs. The test does not allow to make a specific diagnosis of EDs. The questionnaire has been confirmed as a reliable and valid instrument in English [59], Hebrew [60] and Arabic [22,61]. It consists of 26 items scored along a three-point Likert scale (0-3), yielding a possible range of 0-78 points. The EAT-26 includes three subscales dieting, bulimia and food preoccupation (hereafter bulimia); and oral control - as well as a total score. Total scores of 20 or higher indicate elevated body shape and weight concerns and can be used to identify those participants at high risk of developing EDs. In the current study, the entire questionnaire and the dieting subscale had good reliability (Cronbach's alpha $=.891, .834$, respectively) and the bulimia and oral control subscales had acceptable reliability (Cronbach's alpha=.758, .677, respectively). The instrument has been confirmed as a reliable and valid instrument in English [59], Hebrew [60] and Arabic [61].

\section{Procedure}

The complete study protocol was approved by the College Institutional Review Board on 9.12.2019, and the approval number is 3601010170 . All participants signed an informed consent form (the first page of the questionnaire) Completion of the questionnaires in two colleges was voluntary. Participants were told we were interested to know what they thought about some nutrition and family issues. They were promised anonymity and discretion, and were informed that they could stop filling out the questionnaires at any time.

\section{Statistical methods}

Reliability of the measures was assessed by Cronbach's alpha. The assumption of approximate normality was assessed by computation of skewness and kurtosis of the variables of interest.

Missing BMI ( $\mathrm{n}=200)$ was imputed by linear regression (SPSS multiple imputation) using culture, gender and age as predictors. Pooled BMI was used in the statistical analyses.

To examine gender and cultural differences, multivariate two-way ANOVAs were performed to assess the effects of culture and gender on the four metrics of DoS the three EAT-26 subscales and its total score.

In addition, to determine whether there was a relationship between risk of EDs, continuous demographic variables (age and BMI) and DoS, Pearson correlations were performed. Hierarchical regression analyses, with age and gender forced into the model, BMI in the first step and DoS subscales in the second step, were run within each culture to find predictors of the EAT-26 total score and its three subscales in each cultural group separately. All analyses were performed using IBM SPSS (version 24). Significance was set to be $p<.05$.

\section{Results}

\section{Preliminary analyses}

The study variables (of the EAT-26 and the DSI-R) were approximately normally distributed, as skewness and kurtosis were \pm 1.2 . Participants reported total EAT-26 scores of between 0 and 63, with 245 (28.5\%) reporting a positive EAT-26 score (score $\geq 20$ ), indicating high risk of developing EDs. Table 1 presents the high risk of developing EDs by demographic variables. Participants with a high risk of EDs (a positive score) tended to have higher BMI ( $\mathrm{p}<.06$ ) than their counterparts with lower risk of EDs (total score<20). The tendency for positive scores, i.e., a high risk of EDs, was higher for Arabs than for Jews (total score $\geq 20$; $p$.05).

\section{Main findings}


In order to examine the hypotheses 1, 2 regarding cultural and gender differences in the study variables, we ran a series of two-way MANOVAs. Table 2 presents the distribution of DSI-R and EAT-26 scores by culture and gender.

Two-way MANOVAs of the four DSI-R subscales revealed statistically significant main effects of culture $[F(4,851)=17.14, p<.001, p a r t i a l$ eta $=.075]$, gender $[F(4,851)=17.92, p<.001$, partial eta $=.078]$ and the interaction between them $[F(4,851)=2.94, p<.02$, partial eta $=.014]$. Between-participant tests yielded statistically significant cultural effects for I-position and emotional cutoff; gender effects for emotional reactivity, emotional cutoff and fusion with others; and a significant interaction for emotional cutoff (Table 3). Specifically, Jewish participants had higher I-position and lower emotional cutoff than Arabs, while female participants had significantly higher emotional reactivity and fusion with others, and lower emotional cutoff, than their male counterparts. Post-hoc testing of the interaction revealed that Jewish female participants reported statistically significant lower emotional cutoff than Jewish male participants, while there was no significant difference in reported emotional cutoff between Arab males and females. The first and second hypotheses were thus partially supported.

To investigate cultural and gender differences in terms of risk of EDs (Hypotheses 1, 2), we also ran two-way MANOVAs of the EAT-26 subscales. Results revealed a statistically significant main effect of culture $[F(3,852)=4.30, p<.005$, partial eta $=.015]$, as well as a borderline statistically significant main effect of gender $[F(3,852)=2.25, p<.08$, partial eta $=.008]$ and of the interaction between culture and gender $[F(3,852)=2.26, p<.08$, partial eta $=.008]$. Between-participant tests revealed a statistically significant culturaleffect for dieting and bulimia, and a statistically significant gender effect for the dieting subscale. Jewish participants had significantly lower diet and bulimia scores than their Arab counterparts, while female participants had higher dieting scores than male participants. A two-way ANOVA of the total EAT-26 score revealed only a statistically significant main effect of culture $[F(1,854)=12.14, p<.001, p a r t i a l$ eta=.014], with Jewish participants having significantly lower scores than Arab participants (see Table 3).

In order to evaluate whether differentiation of self contributes to the risk of EDs (third hypothesis), Pearson correlations between the DSI-R and EAT-26 subscales, as well as age and BMI, were performed (Table 4). The EAT-26 total score, dieting subscale and bulimia subscale were positively associated with emotional reactivity and emotional cutoff, and negatively associated with I-position. Fusion with others was found positively associated with dieting and bulimia.

Age was negatively correlated with the EAT-26 total score and the oral control subscale, and BMI was positively correlated with the bulimia subscale. Oral control was negatively correlated with I-position and age. The third hypothesis was thus partially corroborated.

Table 5 presents the hierarchical regression. Analyses revealed that, among Jewish participants, BMI was a significant demographic predictor of EAT-26 and emotional reactivity and emotional cutoff were significant DSI-R measures: increased emotional reactivity and increased emotional cutoff increased the EAT26 total score. In contrast, among Arab participants, BMI was not a significant predictor, while I-position and emotional cutoff were significant DSI-R predictors. Increased I-position decreased the EAT-26 total score while increased emotional cutoff increased it. Thus, the hypothesis that the EAT-26 would be negatively associated with DoS was partially confirmed for both Jewish and Arab participants. Comparison of the slopes (beta coefficients) between the Jewish and Arab hierarchical models revealed borderline statistically significant differences in fusion with others [t(839)=1.87, $\mathrm{p}<.06]$ and $\mathrm{I}$-position $[\mathrm{t}(839)=1.86, \mathrm{p}<.06]$. The association between fusion with others and EAT-26 were in opposite directions for Jews (beta=1.541) and Arabs (beta $=-1.421)$. There tended to be a stronger association between I-position and the risk of EDs among Arabs (beta=-2.554) than Jews (beta=-0.503). No statistically significant differences were found in the strength of association between Arabs and Jews in emotional reactivity and emotional cutoff or BMI.

Examination of the three subscales of EAT-26 (Table 6) revealed that, for Jewish participants, BMI was a significant predictor of scores on the bulimia and oral control subscales. Since BMI was imputed, there is missing data. Increased BMI was associated with increased subscale measures. Among Arab participants, BMI predicted the oral control subscale: in contrast to Jewish participants, increased BMI was associated with decreased oral control. With respect to the relation between DoS and the risk of EDs, for Jewish participants, emotional reactivity and emotional cutoff predicted the dieting subscale and emotional cutoff and fusion with others predicted the bulimia subscale; none of the DSI-R components predicted oral control. Among Arab participants, I-position predicted the dieting and bulimia subscales, and emotional cutoff predicted the bulimia and oral control subscales. Emotional cutoff approached significance for the dieting subscale $(p=.07)$.

Comparison of the beta coefficients (via t-tests to compare slopes) from the Arab and Jewish regression models revealed a statistically significant difference in BMI for oral control $[t(853)=3.70, p<.001]$ but not for the bulimia subscale $(t(853)=0.70, p>.48)$. There were no statistically significant differences in DoS beta coefficients between Arabs and Jews for the diet and oral control subscales. For the bulimia subscale, there was a statistically significant difference in Iposition beta coefficients between Jews (beta=.021) and Arabs (beta $=-.170)[t(853)=3.73, p<.001]$.

\section{Discussion}

This study aimed to investigate whether the risk of EDs (measured by EAT-26) is associated with poor DoS (low I-position and high levels of emotional reactivity, emotional cutoff, and fusion with others) and whether results differ by gender and culture. On the whole, results indicated that the risk of EDs was higher among Arabs than Jews and among females than males. The present findings are in line with studies that have indicated a higher risk of EAT-26 among Arabs [62] and among females [57]. Regarding cultural differences, Arabs presented a higher risk of EDs (total EAT-26 score $\geq 20$ ) and higher levels of dieting and bulimia than Jews, supporting a previous study $[20,66]$ and corroborating Hypothesis 1 . One possible reason is that Arab society in Israel is undergoing a process of modernization, which often leads young Arabs to imitate the modern Western majority culture. They are exposed to messages in the media about the culture of thinness and the ideal of beauty and tend to behave in accordance with modern eating and fashion habits [56]. Another reason is that Arabs in Israel are a conservative minority group that still perceives mental health issues, including the risk of EDs, as a stigma accompanied by feelings of shame and discomfort [20]. Since they are less likely to seek treatment in public clinics than Jews, there may be many people who do not receive help and 
treatment for EDs and, therefore, more young adults may suffer from EDs. Another possibility, as suggested by Spivak et al. [25], is that the EAT-26 questionnaire might be perceived differently by different ethnic groups. Future studies are therefore recommended to use an additional suitable questionnaire.

Regarding DoS, Arabs reported lower levels of I-position and higher levels of emotional cutoff, in keeping with previous findings [44] and partially supporting Hypothesis 1 . Arab society is characterized as collectivist, such that the family is highly involved in the lives of its members. In such circumstances, emotional detachment is a useful pattern for coping with daily challenges; emotional cutoff probably helps preserve certain boundaries and allows release. This finding can be likened to the claim that Arab men do not disclose their emotional states and worries due to social expectations in order to preserve a rough and macho appearance [64]; This difficulty in expressing feelings and desires may explain their lower levels of I-position and higher levels of emotional cutoff than Jewish men [65].

The examination of gender differences showed that women reported higher levels of dieting, fusion with others, and emotional reactivity and a lower level of emotional cutoff than men, supporting previous findings $[20,44]$ and partially confirming Hypothesis 2 . An interaction effect was also found: Jewish women reported a lower level of emotional cutoff than Jewish men, whereas no gender differences were found among Arabs.

With regard to the relationship between DoS and the risk of EDs, a low level of I-position and high levels of emotional reactivity and emotional cutoff were found to be associated with a high risk of EDs, partially supporting Hypothesis 3. A growing body of research has suggested that poor DoS is related to a high risk of EDs $[34,66]$. A possible reason for this relationship is based on previous studies showing that eating is not based solely on the mechanism of hunger that signals when and how much to ingest but is also activated by emotional and familial factors [39,41]. Poorly differentiated people have been found likely to feel anxious and distressed more often; some of them may turn to food as a solution to this stress [67].

Our examination of the factors that may be associated with a high risk of EDs among each cultural group yielded interesting results. Among Arabs, high levels of emotional cutoff and low levels of I-position were found associated with a high risk of EDs. Low l-position was found associated with a high risk of dieting and bulimia, and high emotional cutoff was associated with a high risk of bulimia and oral control. Among Jews, high levels of emotional cutoff were found associated with the EAT-26 total score; among females, high emotional reactivity and emotional cutoff were found to be associated with a high risk of dieting, and high emotional cutoff and fusion with others were associated with a high risk of bulimia. It should be noted that, among Arabs only, the indices of DoS were found to be related to oral control. This topic is worthy of further investigation.

The above results partially support earlier findings [37] pointing to associations between the dimensions of DoS and EDs symptoms. Specifically, our results indicate that emotional cutoff may be associated with a high risk of EDs (EAT-26 total score and subscales for Jews and Arabs alike).

However, for Arabs more than Jews, low l-position - i.e., having difficulty expressing one's feelings, needs, and thoughts - was also found correlated with a high risk of EDs. Given that individuals with a lower level of I-position may exhibit poor coping skills in managing stress [38], it is possible that in times of stress, Arabs are more preoccupied with food than Jews. One main cause of EDs may be a lack of communicativeness in stressful interpersonal situations. As suggested, individuals at high risk of EDs may find it difficult to express their emotions and adhere to their feelings and beliefs; this may lead them to seek comfort in food [68].

In contrast, the level of fusion with others was only found positively associated with a high risk of EDs among Jews. The lack of correlation between fusion with others and risk of EDs among Arabs is somewhat surprising given past findings. It seems that very close relationships among Arabs actually provide some support and reduce the risk of EDs. This is perhaps because Arab society is conservative and promotes close relationships rather than perceiving them as disruptive and distressing, as may be the case in Jewish society. Another finding indicates that among Jewish participants, there were positive associations between emotional reactivity and the total score of EAT-26 and the dieting sub-scale.

In conclusion, individuals with high risk of EDs may have difficulty maintaining intimate family relationships. In distressing situations, they tend to disconnect rather than share with or gain support from significant others. Israeli Jews who reported high risk of EDs are those with a higher BMI and a high emotional reactivity which enables them to either disengage from significant others or create fused relationships. Israeli Arabs who reported high risk of ED are those with a higher BMI (positively associated with oral control only), low levels of I-position, and high levels of emotional cutoff. It may indicate that their feelings and needs remain unexpressed.

\section{Limitations, future research, and implications}

This study has several limitations. First, given the cross-sectional nature of the research, any interpretation regarding causality should be made with much caution. Longitudinal studies are needed to provide a more extensive basis for the suggested causal relationships between DoS and the risk of EDs. Second, the study did not examine increased tendencies for unhealthy behaviors, such as improper diet and the avoidance of physical activity. It is strongly recommended that a follow-up study examine these factors. Third, values were not measured at the individual level, under the assumption that Arabs are highly collectivist while Jews are more individualist. Future research should explore this issue further, investigating differences at the individual level within each cultural group. Fourth, we used self-report questionnaires which might have been biased by social desirability. Fifth, the cluster sampling used may have created a bias in the representation of each of the cultures within the wider population. Future studies are recommended to conduct systematic sampling that represents the percentages of each cultural stratum in the population. Sixth, the large percentage of participants whose BMI data was missing, primarily due to omission of their weight, might have biased the results, as our imputation method could not account for any differences between adolescents who were reluctant to provide their weight and those who provided their weight. However, even after removing all those who did not report their weight or eliminating BMI as a variable of interest, we still achieved similar results (not shown). Finally, due to missing data regarding the marriage status of participants' parents and 
those living together but not married, we could not check the differences between those groups. Yet, it should be noted that all the parents of participants included in this study were living together.

Notwithstanding these limitations, the current study supports previous findings association between the risk of Eds and DoS. The findings may have clinical implications in terms of psychological interventions for the treatment and prevention of EDs among young adults in both Western and non-Western societies. Interventions for alleviating EDs should be specifically tailored to address the difficulties associated with low DoS in each cultural group. It is therefore recommended that patients from different cultures are treated differently. Furthermore, we suggest that healthcare providers are aware of the high risk of EDs among young adults and their relationship to DoS in order to educate people about how to share their feelings to a greater extent and improve their communication with family members.

\section{What is already known on this subject?}

Although it is well documented that DoS is a crucial element that may increase susceptibility to certain physical pathologies, there is a paucity of research on its relationship to EDs. Studies examining this topic have looked separately at males [37] and females [46]; however, much less is known about the association between DoS and the risk of EDs (among both genders) in terms of cultural group.

\section{Declarations}

\section{Contributions}

This study supports previous findings regarding the association between DoS and the risk of EDs among women and men living in Israel and belonging to two different cultures. Specifically, the findings suggest that, regardless of culture, those who suffer from a high risk of EDs tend to disconnect from significant others in distressing situations rather than gaining their support. Additionally, Jews who are more vulnerable to the risk of EDs were found to report higher levels of BMI, emotional reactivity, emotional cutoff, and fusion with others, while Arabs who are more vulnerable to the risk of EDs reported higher BMI, higher levels of emotional cutoff, and lower levels of I-position. In light of these results, it is recommended that treatment plans be tailored to each culture.

\section{Compliance with Ethical Standards}

Conflict of interest: The authors declare that they have no conflict of interest.

Ethics approval was given by the college's Committee of Ethics.

Informed consent: All participants signed written informed consent forms prior to taking part in the study.

\section{References}

1. Melisse, B., de Beurs, E., van Furth, E.F. (2020). Eating disorders in the Arab world: A literature review. Journal of Eat Disord 8(1):59. doi: 10.1186/s40337020-00336-x.

2. Thomas JJ, Lee S, Becker AE (2016) Updates in the epidemiology of eating disorders in Asia and the Pacific. Curr Opin Psychiatry 29(6):354-362. doi.org/10.1097/YC0.0000000000000288

3. Lindvall Dahlgren C, Wisting L, Rø $\varnothing(2017)$ Feeding and eating disorders in the DSM-5 era: A systematic review of prevalence rates in non-clinical male and female samples. Journal of Eating Disorders 5(1):1-10. https://doi.org/10.1186/s40337-017-0186-7

4. Mitchison D, Hay PJ (2014) The epidemiology of eating disorders: Genetic, environmental, and societal factors. Clinical Epidemiology 17:89-97. doi: 10.2147/CLEP.S40841.

5. Smink FR, van Hoeken D, Hoek HW (2013) Epidemiology, course, and outcome of eating disorders. Curr Opin Psychiatry 26(6):543-548. doi: 10.1097/YCO.0b013e328365a24f.

6. Smink FR, van Hoeken D, Oldehinkel AJ, Hoek HW (2014) Prevalence and severity of DSM-V eating disorders in a community cohort of adolescents. International J of Eating Disorders 47(6):610-619. doi: 10.1002/eat.22316.

7. Udo T, Grilo CM (2018) Prevalence and correlates of DSM-5-defined eating disorders in nationally representative sample of U.S. adults. Biological Psychiatry 84(5):345-354. doi: 10.1016/j.biopsych.2018.03.014.

8. Latzer Y, Tzischinsky O, Azaiza F (2013) Not just a Western girls' problem: Eating attitudes among Israeli-Arab adolescent boys and girls. International Journal of Adolescence and Youth 19(3):382-394. doi.org/10.1080/02673843.2012.747973

9. Phillips L, Kemppainen JK, Mechling BM, Mackain S, Kim-Godwin Y, et al (2015) Eating disorders and spirituality in college students. J Psychosoc Nurs Ment Health Serv 53(1):30-37. doi.org/10.3928/02793695-20141201-01

10. Chan YL, Samy AL, Tong WT, Islam MA, Low WY (2020) Eating disorder among Malaysian university students and its associated factors. Asia Pac $J$ Public Health 32(6-7):334-339. doi: 10.1177/1010539520947879.

11. Ngan SW, Chern BCK, Rajarathnam DD, Balan J, Hong TS, Tiang KP (2017) The relationship between eating disorders and stress among medical undergraduate: A cross-sectional study. Open J Epidemiol 7:85-95. doi.org/10.4236/ojepi.2017.72008 
12. Pengpid S, Peltzer K (2018) Risk of disordered eating attitudes and its relation to mental health among university students in ASEAN. Eat Weight Disord 23:349-355. doi.org/10.1007/s40519-018-0507-0

13. Azzouzi N, Ahid S, Bragazzi NL, Aarab C, et al (2019) Eating disorders among Moroccan medical students: Cognition and behavior. Psychol Res Behav Manag 12:129-135. doi.org/10.2147/PRBM.S165114

14. Abd El-Azeem Taha AA, Abu-Zaid HA, El-Sayed Desouky D (2018) Eating disorders among female students of Taif University, Saudi Arabia. Arch Iran Med 21(3):111-117.

15. Hoek HW (2016) Review of the worldwide epidemiology of eating disorders. Current Opinion in Psychiatry 29(6):336-339. doi.org/10.1097/YCO.0000000000000282

16. Smink FR, van Hoeken D, \& Hoek HW (2012) Epidemiology of eating disorders: Incidence, prevalence and mortality rates. Current Psychiatry Reports 14(4):406-414. doi.org/10.1007/s11920-012-0282-y

17. Makino M, Tsuboi K, Dennerstein L (2004) Prevalence of eating disorders: A comparison of Western and non-Western countries. Medscape J Gen Med 6(3):49.

18. Musaiger AO, Al-Mannai M, Tayyem R, Al-Lalla O, Ali EYA, Kalam F, et al (2013) Risk of disordered eating attitudes among adolescents in seven Arab countries by gender and obesity. A cross-cultural study. Appetite 60(1):162-167.

19. Feinson MC, Meir A (2014) Disordered eating \& cultural diversity: A focus on Arab Muslim women in Israel. Eating Behavior 15(2):306-310. doi: 10.1016/j.eatbeh.2014.03.004.

20. Latzer Y, Azaiza F, Tzischinsky O (2009) Eating attitudes and dieting behavior among religious subgroups of Israeli-Arab adolescent females. Journal of Religious Health 48(2):189-199. doi: 10.1007/s10943-008-9189-7.

21. Latzer Y, Tzischinsky O, Azaiza F (2007) Disordered eating related behaviors among Arab schoolgirls in Israel: An epidemiological study. International J of Eating Disorders 40:263-270. doi.org/10.1002/eat.20348

22. Latzer Y, Tzischinsky O, Geraisy N (2007) Comparative study of eating-related attitudes and psychological traits between Israeli-Arab and -Jewish school girls. J of Adolescence 30(4):627-637.

23. Latzer Y, Tzischinsky O (2005) Eating attitudes in a diverse sample of Israeli adolescent females: A comparison study. J of Adolescence 28(3):317-323. doi.org/10.1016/j.adolescence.2004.12.004

24. Levinson CA, Rodebaugh TL (2016) Clarifying the prospective relationships between social anxiety and eating disorder symptoms and underlying vulnerabilities. Appetite 1 (107):38-46. https://doi.org/10.1016/j.appet.2016.07.024

25. Spivak-Lavi Z, Peleg O, Tzischinsky O, Stein D, Latzer Y (2021) Differences in the factor structure of the Eating Attitude Test-26 (EAT-26) in different cultures in Israel: Jews, Muslims, and Christians. Nutrients 13(6): 1899. https://doi.org/10.3390/nu13061899

26. Israel Central Bureau of Statistics (2018) Statistical abstract of Israel. Jerusalem: ICBS.

27. Fenison MC, \& Meir A (2014) Disordered eating \& cultural diversity: a focus on Arab Muslim women in Israel. Eat Behav. 15(2): 306-10. doi: 10.1016/j.eatbeh.2014.03.004.

28. Musaiger AO, Al-Kandari FI, Al-Mannai M, Al-Faraj AM, Bouriki FA, Shehab FS, Al-Dabous LA, Al-Qalaf WB (2016) Disordered eating attitudes among university students in Kuwait: The role of gender and obesity. International Journal of Preventive Medicine 7(1):67. doi: 10.4103/2008-7802.180413.

29. Harel Y, Ellenbogen-Frankovits S, Molcho M, Abu-Ashas K, Habib J (2002) Youth in Israel. Brookdale Institute, Jerusalem.

30. Latzer, Y, Tzischinsky, O, Spivak-Lavie, Z, Chen, I, Weinberger-Litman S (2018) The relationship between disordered eating pathology, sense of coherence and body image among adolescent boys in Israel. Israel J. Psychiatry 55(1): 66-72

31. Schaumberg K, Jangmo A, Thornton LM, Birgegård L, Almqvist C, Claes Norring C, Larsson H, BulikCM (2019) Patterns of diagnostic transition in eating disorders: A longitudinal population study in Sweden. Psychol Med 49(5):819-827. DOI: 10.1017/S0033291718001472.

32. Gutiérrez T, Espinoza P, Penelo E, Mora M, González ML, Rosés R, Raich RM (2015) Association of biological, psychological and lifestyle risk factors for eating disturbances in adolescents. Journal of Health Psychology20(6):839-849. doi: 10.1177/1359105315577302.

33. Le Grange D, Lock J, Loe K, \& Nicholls D (2010) Academy for eating disorders position paper: The role of the family in eating disorders. International Journal of Eating Disorders, 43(1): 1-5. https://doi.org/10.1002/eat.20751

34. Doba K, Berna G, Constant E, Nandrino JL(2018) Self-differentiation and eating disorders in early and middle adolescence: A cross-sectional path analysis. Eating Behavior29:75-82. doi: 10.1016/j.eatbeh.2018.03.003.

35. Radwan H, Hayder A, Hasan HA, Najm L, Zaurub S, Jami F, Javadi F, Deeb LA, Iskandarani A (2018) Eating disorders and body image concerns as influenced by family and media among university students in Sharjah, UAE. Asia Pac J Clin Nutr 27(3):695-700 https://doi.org/10.6133/apjcn.062017.10

36. Blackmore V, Searight H, Ratwik SH (2011) The relationship between eating attitudes, body image and perceived family-of-origin climate among college athletes. North American Journal of Psychology 13:435-446.

37. Buser JK, Gibson S (2014) Differentiation and eating disorder symptoms among males. The Family Journal: Counseling \& Therapy for Couples and Families 22(1):17-25. doi.org/10.1177/1066480713504903

38. Kerr ME, Bowen M (1988) Family evaluation: An approach based on Bowen theory. New York: Norton.

39. Skowron EA, Friedlander M (1998) The Differentiation of Self Inventory: Development and initial validation. Journal of Counseling Psychology28:235-246. doi.org/10.1037/a0016709

40. Skowron EA (2000) The role of differentiation of self in marital adjustment. Journal of Counseling Psychology 47:229-237. doi.org/10.1037/00220167.47.2.229 
41. Peleg O, Tzischinsky O (2016) Assessing satisfaction with differentiation of self through circle drawing: Validation of a revised self-report instrument (SFIR). Journal of Psychology \& Psychotherapy5:1-10. DOI: 10.4172/2161-0487.1000228. DOI: 10.4172/2161-0487.1000228

42. Skowron EA, Schmitt TA (2003) Assessing interpersonal fusion: Reliability and validity of a new DSI Fusion with Others subscale. Journal of Marital and Family Therapy 29:209-222. doi.org/10.1111/j.1752-0606.2003.tb01201.x

43. Peleg O, Halaby E, Whaby E (2006) The relationship of maternal separation anxiety and differentiation of self to children's separation anxiety and adjustment to kindergarten: A study in Druze families. Journal of Anxiety Disorders 20:973-995. doi: 10.1016/j.janxdis.2006.01.008. Epub 2006 Feb 23.

44. Peleg O, Zoabi M (2014) Social anxiety and differentiation of self: A comparison of Jewish and Arab college students. Pers Indiv Differ 68:221-228. https://doi.org/10.1016/j.paid.2014.04.032

45. Scigala DK, Fabris LMA, Badenes-Ribera E, Zdankiewicz-Scigala CL (2021) Alexithymia and self differentiation: The role of fear of intimacy and insecure adult attachment. Contemp Fam Ther 43:165-176. https://doi.org/10.1007/s10591-021-09567-9

46. Skowron EA, Stanley KL, Shapiro MD (2009) A longitudinal perspective on differentiation of self, interpersonal and psychological well-being in young adulthood. Contemporary Family Therapy 31(1):3-18. https://doi.org/10.1007/s10591-008-9075-1

47. Cohen A, Peleg O, Sarhana A, Lam S, Haimov I (2019) Psychological, educational and familial dimensions among diabetic adolescents and adults. International Journal of Behavioral Medicine. https://doi.org/10.1007/s12529-019-09816-0.

48. Manzi CV, Vignoles VL, Regalia C, Scabini E (2006) Cohesion and enmeshment revisited: Differentiation, identity, and well-being in two European cultures. Journal of Marriage and Family 68:673-689. https://doi.org/10.1111/j.1741-3737.2006.00282.x

49. Peleg O, Yaniv I, Katz R, Tzischinsky O (2018) Does trait anxiety mediate the relationship between family patterns and quality of life? The American Journal of Family Therapy 46(4):341-355. doi.org/10.1080/01926187.2018.1540282

50. Doba K, Nandrino J-L, Dodin V, Antoine P (2014) Is there a family profile of addictive behaviors? Family functioning in anorexia nervosa and drug dependence disorder. Journal of Clinical Psychology 70(1):107-117. DOI: 10.1002/jclp.21977

51. Rothschild-Yakar L, Bashan-Levi Y, Gur E, Vorgaft I, Stein D (2015). Dependent and autonomous interpersonal styles and level of differentiation among male patients with eating disorders. Psychoanalytic Psychology 33:243-263. doi.org/10.1037/pap0000026

52. Pallister E, Waller G (2008) Anxiety in the eating disorders: Understanding the overlap. Clin Psychol Rev 28(3):366-386. DOI: 10.1016/j.cpr.2007.07.001

53. Torres S, Guerra MP, Lencastre L, Miller K, Vieira FM, Roma-Torres A, Costa P (2015) Alexithymia in anorexia nervosa: The mediating role of depression. Psychiatry Research 225:99-107. doi.org/10.1016/j.psychres.2014.10.023

54. Hooper LM, Doehler K (2011) The mediating and moderating effects of differentiation of self on body mass index and depressive symptomatology among an American college sample. Counselling Psychology Quarterly 24(1):71-82. doi.org/10.1080/09515070.2011.559957

55. Peleg 0, Tzischinsky 0, Spivak-Lavi Z (In Press) Does depression mediate the relationship between differentiation parenting styles and eating disorders? International Journal of Psychology.

56. Latzer Y, Weinberger-Litman S, Spivak-Lavi Z, Tzischinsky O (2019) Disordered eating pathology and body image among adolescent girls in Israel: The role of sense of coherence. Community Mental Health Journal:1-7. DOI: 10.1007/s10597-019-00446-0 [IF 1.460].

57. Peleg-Popko O (2002) Bowen theory: A study of differentiation of self and students' social anxiety and physiological symptoms. Contemporary Family Therapy24:355-369. doi.org/10.1023/A:1015355509866

58. Peleg 0 (2008) The relation of differentiation of self and marital satisfaction: What can be learned from married people over the life course? The American Journal of Family Therapy36:1-14. doi.org/10.1080/01926180701804634

59. Garner DM, Olmsted MP, Bohr Y, Garfinkel PE (1982) The Eating Attitudes Test: Psychometric features and clinical correlates. Psychological Medicine 12:871-878. DOI: $10.1017 /$ s0033291700049163

60. Ianuca I (1990) Validation of the EAT in Israel (doctoral dissertation). School of Medicine; Tel Aviv University, Israel.

61. Al-Subaie A, al-Shammari S, Bamgboye E, al-Sabhan K, al-Shehri S, Bannah AR (1996) Validity of the Arabic version of the Eating Attitude Test. International Journal of Eating Disorders 20:321-324. DOI: 10.1002/(SICI)1098-108X(199611)20:3<321::AID-EAT12>3.0.C0;2-2

62. Fatima W, Ahmad LM (2018) Prevalence of disordered eating attitudes among adolescent girls in Arar City, Kingdom of Saudi Arabia. Health Psychology Research 29(1):7444. doi: 10.4081/hpr.2018.7444.

63. Terhoeven V, Nikendei C, Bärnighausen T, Bountogo M, Friederich HC, Ouermi L, Sié A, Harling G (2019) Eating disorders, body image and media exposure among adolescent girls in rural Burkina Faso. Tropical Medicine \& International Health 11. doi: 10.1111/tmi.13340.

64. Dwairy M, Achoui M (2010) Adolescent-family connectedness: A first cross-cultural research on parenting, culture and psychological adjustment of children. Journal of Child and Family Studies 19:16-22. doi.org/10.1007/s10826-009-9335-1

65. Dwairy M (2019) Culture and leadership: Personal and alternating values within inconsistent cultures. International Journal of Leadership in Education: Theory and Practice 22:510-518. doi.org/10.1080/13603124.2017.1394498

66. Tasca GA, Demidenko N, Krysanski V, Bissada H, Illing V, Gick M, Weekes K, Balfour L (2009) Personality dimensions among women with an eating disorder: Towards reconceptualizing DSM. European Eating Disorders Review 17(4):281-289. doi: 10.1002/erv.938.

67. Tsenkova VK, Karlamangla A (2016) Depression amplifies the influence of central obesity on 10-year incidence of diabetes: Findings from MIDUS. PLoS One11(10):e0164802. doi: 10.1371/journal.pone.0164802

68. Ruah M, Peleg $O$ (2019) The relationships between differentiation of self, birth order and separation anxiety: Comparison between Jewish and Arab students. The School Counselor 21: 195-220 (Hebrew). 
Tables

Table 1 Demographic characteristics by risk of developing eating disorders, $t$-test and $\mathrm{X}^{2}$ between groups $(\mathrm{N}=859)$

\begin{tabular}{llllll} 
& $\begin{array}{l}\text { Positive EAT-26 score }(\geq 20) \\
(\mathrm{N}=245)\end{array}$ & $\begin{array}{l}\text { Negative EAT-26 score }(<20) \\
(\mathrm{N}=614)\end{array}$ & $\mathrm{t}$ & $\mathrm{p}$ \\
\hline Mean $(\mathrm{SD})$ & Mean $(\mathrm{SD})$ & & \\
\hline Age & $26.3(5.5)$ & $27.0(5.6)$ & 1.63 & .09 \\
\hline BMI & $24.36(4.67)$ & $23.60(4.13)$ & -2.16 & .04 \\
\hline Imputed BMI & $24.19(4.67)$ & $26.99(4.47)$ & -1.92 & .06 \\
\hline Gender & $\mathrm{N}(\%)$ & $\mathrm{N}(\%)$ & $\mathrm{X}^{2}$ & \\
\hline Male & $50(26.5 \%)$ & & .50 & \\
\hline Female & $195(29.1 \%)$ & $139(73.5 \%)$ & & \\
\hline Culture & & $475(70.9 \%)$ & & \\
\hline Jew & $107(24.3 \%)$ & & & & \\
\hline Arab & $138(32.9 \%)$ & $281(67.1 \%)$ & & \\
\hline
\end{tabular}

Table 2 Means, standard deviations and ranges for differentiation of self and risk of eating disorders by gender and culture $(\mathrm{N}=859)$

Table 3 Culture and gender differences in risk of eating disorders: Values of $\mathrm{F}$ for analyses of variance (two-way ANOVAs)

\begin{tabular}{|c|c|c|c|c|c|c|c|c|c|}
\hline & \multicolumn{3}{|l|}{ Culture } & \multicolumn{3}{|l|}{ Gender } & \multicolumn{3}{|c|}{ Culture*Gender } \\
\hline & $F(1,854)$ & $\mathrm{p}$ & Partial & $F(1,854)$ & $\mathrm{p}$ & Partial & $F(1,854)$ & $\mathrm{p}$ & Partial \\
\hline & & & eta $^{2}$ & & & eta $^{2}$ & & & eta $^{2}$ \\
\hline \multicolumn{10}{|l|}{ DSI-R } \\
\hline Emotional reactivity & 2.09 & .15 & .002 & 25.88 & .001 & .029 & 0.00 & .98 & .000 \\
\hline I-position & 13.89 & .001 & .016 & 0.02 & .90 & .000 & 0.31 & .58 & .000 \\
\hline Emotional cutoff & 27.61 & .001 & .031 & 9.81 & .002 & .011 & 8.60 & .003 & .010 \\
\hline Fusion with others & 0.02 & .89 & .000 & 20.15 & .001 & .023 & 0.46 & .50 & .001 \\
\hline \multicolumn{10}{|l|}{ EAT-26 } \\
\hline Dieting & 11.17 & .001 & .013 & 5.42 & .02 & .006 & 0.99 & .32 & .001 \\
\hline Bulimia & 10.26 & .001 & .012 & 0.73 & .39 & .001 & 2.35 & .12 & .003 \\
\hline Oral control & 2.18 & .14 & .003 & 0.07 & .79 & .000 & 1.22 & .27 & .001 \\
\hline Total & 12.14 & .001 & .014 & 2.10 & .15 & .002 & 0.43 & .51 & .001 \\
\hline
\end{tabular}

Table 4 Pearson correlations of the EAT-26 (total and subscales) with DSI-R subscales, age and BMI (N=859)

\begin{tabular}{lccll} 
& Total & Dieting & Bulimia & Oral control \\
\hline Emotional reactivity & $.126^{\star \star}$ & $.150^{\star \star}$ & $.107^{\star}$ & .036 \\
\hline I-position & $-.172^{\star *}$ & $-.160^{\star *}$ & $-.151^{* \star}$ & $-.104^{*}$ \\
\hline Emotional cutoff & $.165^{\star \star}$ & $.147^{\star \star}$ & $.178^{\star \star}$ & .085 \\
\hline Fusion with others & .084 & $.099^{\star}$ & $.100^{\star}$ & .003 \\
\hline Age & $-.088^{\star}$ & -.073 & -.025 & $-.100^{\star}$ \\
\hline BMI $^{1}$ & .039 & .050 & $.104^{\star}$ & -.057
\end{tabular}

${ }^{1}$ pooled ${ }^{*} p<.01{ }^{* *} p<.001$ 


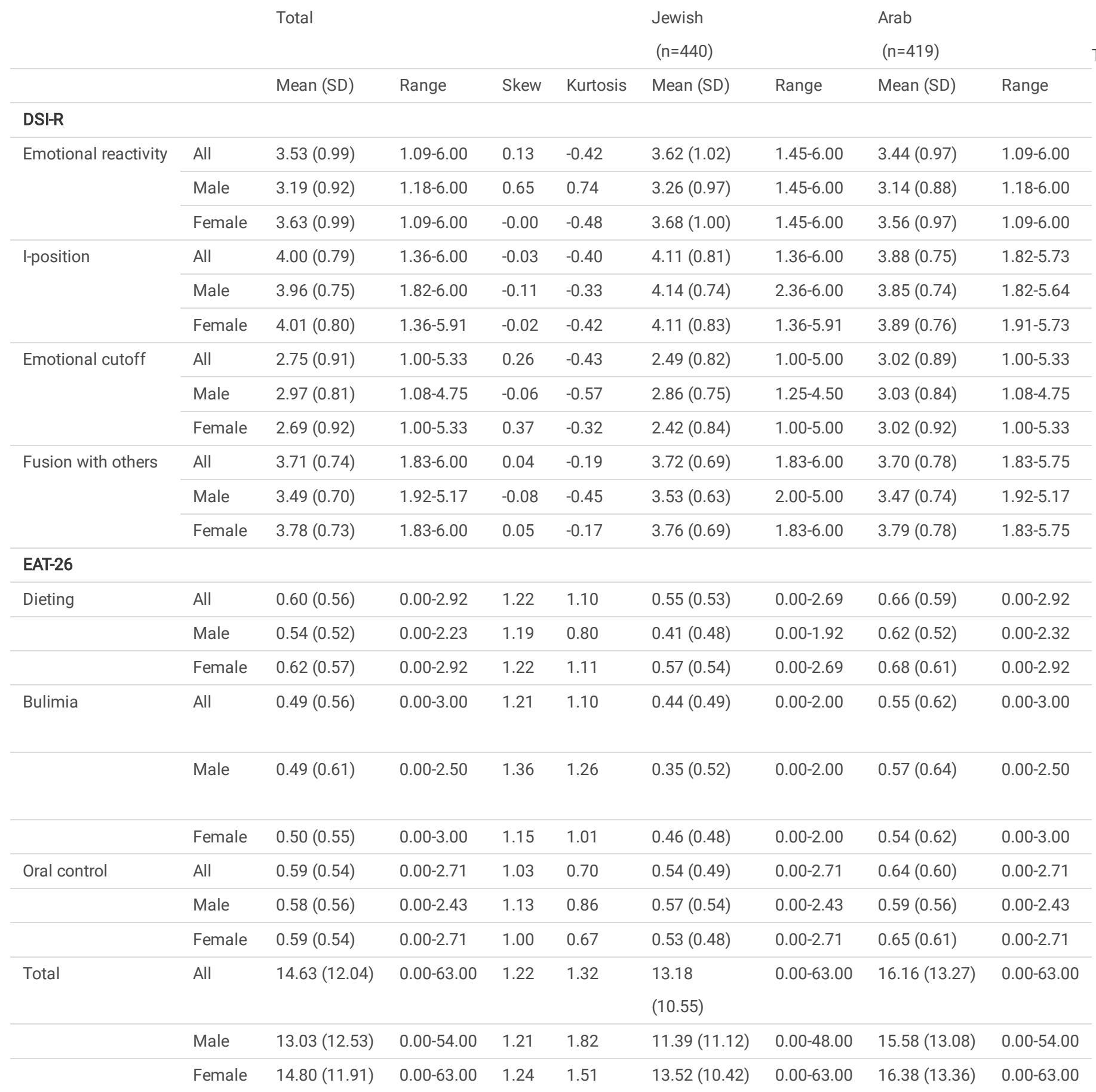

Hierarchical regression analyses with DSI-R subscales as independent variables and total EAT-26 score as dependent variable 
Total EAT-26

\begin{tabular}{|c|c|c|c|c|c|c|c|c|}
\hline \multirow[b]{2}{*}{ Demographics } & \multicolumn{4}{|c|}{ Jewish $(\mathrm{N}=440)$} & \multicolumn{4}{|c|}{$\operatorname{Arab}(\mathrm{N}=419)$} \\
\hline & Beta & SE & $\mathrm{p}$ & partial $\mathrm{R}^{2}$ & Beta & SE & $\mathrm{p}$ & partial $R^{2}$ \\
\hline Age & -.133 & .103 & .196 & - & -.244 & .124 & .05 & - \\
\hline Gender & 2.707 & 1.384 & .05 & - & 1.026 & 1.49 & .49 & - \\
\hline $\mathrm{BMI}^{*}$ & .318 & .131 & .018 & .020 & .066 & .195 & .74 & .001 \\
\hline \multicolumn{9}{|l|}{ DSI-R } \\
\hline Emotional reactivity & 1.4031 & .682 & .04 & .068 & -0.083 & 1.138 & .94 & .000 \\
\hline I-position & -0.503 & .688 & .461 & .000 & -2.554 & .865 & .003 & .016 \\
\hline Emotional cutoff & 1.590 & .628 & .01 & .016 & 2.324 & .918 & .01 & .012 \\
\hline Fusion with others & 1.541 & 0.954 & .11 & .006 & -1.421 & 1.255 & .26 & .002 \\
\hline \multicolumn{9}{|l|}{ Model } \\
\hline $\mathrm{F}$ & & 8.14 & & & & 2.81 & & \\
\hline Adj $R^{2}$ & & .091 & & & & .029 & & \\
\hline $\mathrm{p}$ & & .001 & & & & .007 & & \\
\hline
\end{tabular}

Note: *pooled BMI

Table 6 Hierarchical regression analyses with DSI-R subscales as independent variables and EAT-26 subscales as dependent variable

\begin{tabular}{|c|c|c|c|c|c|c|c|c|c|c|c|c|c|c|c|c|c|c|c|}
\hline \multirow[b]{3}{*}{$\begin{array}{l}\text { Demo- } \\
\text { graphics }\end{array}$} & \multicolumn{8}{|c|}{ Dieting } & \multicolumn{8}{|c|}{ Bulimia } & \multicolumn{3}{|c|}{ Oral control } \\
\hline & \multicolumn{4}{|c|}{ Jewish $(\mathrm{N}=440)$} & \multicolumn{4}{|c|}{$\operatorname{Arab}(\mathrm{N}=419)$} & \multicolumn{4}{|c|}{ Jewish $(\mathrm{N}=440)$} & \multicolumn{4}{|c|}{ Arab $(\mathrm{N}=419)$} & \multicolumn{3}{|c|}{ Jewish $(\mathrm{N}=440)$} \\
\hline & Beta & SE & $\mathrm{p}$ & $\begin{array}{l}\text { Part } \\
\mathrm{R}^{2}\end{array}$ & Beta & SE & $\mathrm{p}$ & $\begin{array}{l}\text { Part } \\
\mathrm{R}^{2}\end{array}$ & Beta & SE & $\mathrm{p}$ & $\begin{array}{l}\text { Part } \\
\mathrm{R}^{2}\end{array}$ & Beta & SE & $\mathrm{p}$ & $\begin{array}{l}\text { Part } \\
\mathrm{R}^{2}\end{array}$ & Beta & SE & $\mathrm{p}$ \\
\hline Age & -.008 & .005 & .13 & - & -.010 & .006 & .08 & - & -.003 & .005 & .62 & - & -.003 & .005 & .58 & - & .002 & .005 & .60 \\
\hline Gender & .174 & .070 & .01 & - & .091 & .067 & .17 & - & .136 & .64 & .03 & - & -.031 & .068 & .64 & - & -.018 & .063 & .78 \\
\hline BMI & .010 & .007 & .14 & - & .013 & .010 & .22 & - & .019 & .007 & .01 & .033 & .011 & .009 & .21 & .007 & .012 & .006 & .03 \\
\hline \multicolumn{20}{|l|}{ DSI-R } \\
\hline ER & .069 & .035 & .05 & .059 & .045 & .051 & .38 & .004 & .025 & .032 & .43 & .001 & -.055 & .053 & .30 & .000 & .057 & .032 & .08 \\
\hline IP & -.035 & .035 & .31 & .002 & -.095 & .038 & .01 & .013 & .021 & .032 & .50 & .001 & -.170 & .040 & .001 & .029 & -.027 & .033 & .40 \\
\hline EC & .063 & .032 & .05 & .011 & .075 & .041 & .07 & .012 & .093 & .029 & .002 & .040 & .153 & .043 & .001 & .033 & .033 & .030 & .26 \\
\hline FO & .064 & .049 & .19 & .005 & -.082 & .056 & .14 & .009 & .099 & .045 & .03 & .021 & -.009 & .058 & .87 & .000 & .023 & .045 & .61 \\
\hline \multicolumn{20}{|l|}{ Model } \\
\hline $\mathrm{F}$ & & 6.72 & & & 2.60 & & & & 7.31 & & & & 4.70 & & & & & 3.46 & \\
\hline Adj $R^{2}$ & & .076 & & & .026 & & & & .091 & & & & .063 & & & & & .037 & \\
\hline$p$ & & .001 & & & .01 & & & & .001 & & & & .001 & & & & & .001 & \\
\hline
\end{tabular}

Note: $\mathrm{ER}=$ emotional reactivity; IP=I-position; $\mathrm{EC}=$ emotional cutoff; $\mathrm{FO}=$ fusion with others. 